\title{
Dorsal Column Stimulation Treats Pain from Chemotherapy-induced, Small Fiber Neuropathy
}

\author{
John P. Kenny, MD and David M. Dickerson, MD
}

Small fiber neuropathy (SFN) is a disorder of small afferent nerve fibers that can result in debilitating pain and functional limitations. There are many etiologies including, but not limited to, diabetes, vitamin deficiencies, infections, and exposure to neurotoxic drugs such as chemotherapeutics. The constellation of signs and symptoms overlap with other disease states leading to potential misdiagnosis. New tests including histologic studies of skin biopsies and autonomic nerve tests have emerged in the last 20 years improving differentiation between these disease processes and SFN.

Multiple chemotherapeutic medications have been implicated in causing SFN, including vincristine which was the causative agent in this case report. The exact incidence of chemotherapy-induced peripheral neurotoxicity (CIPN) is currently unknown, but according to some publications it has been reported to be as high as $40 \%$ in patients that have been treated with chemotherapy. As the number of cancer survivors continues to grow, the number of patients with painful SFN will potentially increase.

Devising an effective analgesic regimen for patients with painful SFN can be difficult, and often requires the pain physician to employ multiple pharmacologic and non-pharmacologic therapies. Treatments include analgesics from several drug classes: antidepressants, opioids, and anticonvulsants. Often times however, more advanced interventional techniques must be employed as effective pain control may be limited by medication side effects or inadequate return of function. While dorsal column stimulation was approved for a limited number of applications, a number of new applications are reported in the literature. In this paper, we present a case of vincristineinduced SFN successfully treated with neuromodulation via spinal cord stimulator.

Key words: Vincristine, vinblastine, chemotherapy-induced neuropathy, chronic pain, spinal cord stimulation, dorsal column stimulation, neuromodulation, small fiber neuropathy, neuropathic pain
Peripheral neuropathy affects between 10 and 20 million Americans over the age of 40 (1). Small fiber neuropathy (SFN), a subset of peripheral neuropathy, is characterized by damage to small somatic or autonomic fibers, or, in some cases, both. Of the peripheral neuropathies, SFN accounts for $3 \%$, the

From: University of Chicago Medicine, Chicago, IL

Author for correspondence: David M. Dickerson, MD

Address: University of Chicago Medicine, Department of Anesthesia \& Critical Care, 5841 S. Maryland Ave MC4028, O-416, Chicago, IL 60637 E-mail: ddickerson@dacc.uchicago.edu majority of which are attributed to diabetes (2). Peripheral nerve fibers are classified by size and presence of myelination. Small fibers include myelinated A-delta fibers and unmyelinated C-fibers (3). Table 1 describes common causes of SFN.

Sensory neuropathy is manifest by painful paresthesias and abnormal findings of small fiber function on at least one of the following: neurologic examination, specialized electrodiagnostic testing, or pathologic studies (4). An alternative diagnosis must be considered if there is evidence of large-fiber dysfunction. Evidence of large-fiber dysfunction includes decreased toe proprioception, decreased vibratory sense at or 
Table 1. Causes of small fiber neuropathy.

\begin{tabular}{|l|l|}
\hline Metabolic conditions & $\begin{array}{l}\text { Diabetes } \\
\text { Hyperlipidemia }\end{array}$ \\
\hline Toxic effects & $\begin{array}{l}\text { Alcohol abuse } \\
\text { Heavy metals } \\
\text { Chemotherapeutics }\end{array}$ \\
\hline $\begin{array}{l}\text { Deficiency or excess of } \\
\text { vitamins }\end{array}$ & Vitamin B12 \\
\hline Infections & $\begin{array}{l}\text { Hepatitis C } \\
\text { Lyme disease } \\
\text { Human immunodeficiency virus } \\
\text { (HIV) } \\
\text { Leprosy }\end{array}$ \\
\hline Auto-immune conditions & $\begin{array}{l}\text { Vasculitis } \\
\text { Paraneoplastic conditions } \\
\text { Sjögren's syndrome } \\
\text { Celiac disease }\end{array}$ \\
\hline Hematologic conditions & Paraproteinemia \\
\hline Hereditary neuropathies & $\begin{array}{l}\text { Fabry disease: alpha- } \\
\text { galactosidase A enzyme } \\
\text { deficiency } \\
\text { Familial amyloidosis: } \\
\text { transthyretin gene mutation }\end{array}$ \\
\hline
\end{tabular}

above the ankles, distal wasting or weakness, generalized areflexia, or abnormal findings on routine nerve conduction studies or needle electromyography (5). Some patients exhibit loss of vibratory sensation at the toes, absent ankle reflexes, or both. Indicators of large-fiber dysfunction are exclusionary, specifically, decreased proprioception at the toes and vibratory loss at or above the ankles.

Symptoms typically start with burning pain in the feet and numbness in the toes (3). Symptoms often begin distally in a length-dependent fashion (6). Symptoms are described as tingling, burning, prickling, shooting, or aching. They often occur at night and interfere with sleep. Not all patients exhibit these dysesthesias; some have numbness and sensation of cold or tightness (4). SFN also can present as restless leg syndrome (7). Autonomic symptoms such as flushing, sweating, skin discoloration, xerostomia, xerophthalmia, or changes in skin temperature can be present as well as gastrointestinal, bladder, or sexual function disturbances (8).

A diagnosis of SFN should be suspected in patients with the symptoms described above and accompanying normal nerve conduction studies. Other auto- nomic tests, quantitative sensory testing, or analysis of epidermal nerve fiber density can be performed (4). Among the autonomic tests are the quantitative sudomotor axon test (QSART), the thermoregulatory sweat test (TST), and cardiovascular response tests.

QSART assesses postganglionic sympathetic cholinergic sudomotor function by stimulating sweat glands through acetylcholine iontophoresis and then measuring evaporated sweat. In the TST, sweating is stimulated in patients in a warming cabinet. Alizaprin powder on the body changes color when in contact with sweat. Cardiovascular response to deep breathing, to valsalva, or to table tilt may also be evaluated. Because the sensitivities of the individual tests are low, a combination of one or 2 sudomotor and 2 or 3 cardiovascular tests is performed (9).

Treatment is targeted towards controlling the cause and then treating the pain. Recommendations for pharmacologic interventions have been published (10). For localized neuropathic pain topical lidocaine is recommended; for generalized pain, oral analgesics are advocated. First line treatment includes tricyclic antidepressants, gabapentinoids, and selective serotonin and norepinephrine reuptake inhibitors are recommended. For second line treatment, opioids and tramadol are recommended. For third line treatment, bupropion, selective serotonin reuptake inhibitors, and anticonvulsants are recommended. Lastly dextromethorphan, memantine, or topical capsaicin are used for generalized pain refractory to the previous lines of therapy.

Non-pharmacologic interventions also have been proven effective. Applying a hot or cold compress has been helpful in some patients (8). Pain relief may come with massage, limb elevation or lowering, skin moisturizers, exercise, and physical or psychological therapy. Spinal cord stimulators and intrathecal morphine are helpful in a select group of patients such as the patient described in this case report (10).

\section{CASE PRESENTATION}

A 24-year-old man presented to the pain clinic for chronic bilateral leg pain. The pain started when he was 16 years old after vincristine chemotherapy treatment for stage IV non-Hodgkin's lymphoma. The patient's weight fluctuated dramatically during his prolonged treatment. Initially the pain was mild 
and intermittent, although over time it had become more severe and debilitating. The bilateral thigh pain had extended to his feet, producing a sensation of pins and needles, and was associated with allodynia, weakness, and decreased proprioception. Symptoms worsened with activity and prevented his ability to stand for more than 15 minutes or walk a city block without the urgent need to sit due to feeling his legs would give out. The pain disrupted his sleep and personal relationships and limited his ability to work outside of the home.

The patient tried pregabalin, topiramate, amitriptyline, topical local anesthetics, nonsteroidal antiinflammatory drugs (NSAIDs), and opioids experiencing significant sedation and cognitive blunting without pain relief. Additionally, he underwent pharmacologic interventions including physical therapy, acupuncture, and massage therapy, again, with minimal relief. Upon establishing a new primary care physician, he was referred to the pain clinic. At this time, the patient was not taking any analgesics due to previous medication-related cognitive side effects and lack of relief.

The physical examination revealed no defects in reflexes, motor function, fine touch, or vibratory sense in the lower extremities. Electromyography and bilateral sural sensory nerve action potentials were normal. The left tibial and right peroneal motor responses and $F$ wave latencies were normal. A needle study of selected muscles of the right lower extremity and right lumbar paraspinal muscles was unremarkable. Lumbar and thoracic magnetic resonance imaging (MRI) was without abnormality. As his pain persisted despite his previous exposure to comprehensive pharmacologic and nonpharmacologic therapy, a trial of spinal cord stimulation was initiated. At this time the patient had been living with this pain and its functional impairment for 7 years.

A dual, compact, octet-lead trial was a success with $>75 \%$ relief of pain symptoms with the central point of stimulation being identified at the top of the T11 vertebral body. Numeric rating score reduced from an 8 (of 10 severity) to a $1-2$ severity. In addition to pain relief, he increased function and perceived strength increased, allodynia decreased, sleep improved, and perceived focus and concentration improved. A Medtronic dual $60 \mathrm{~cm}$, compact, octet lead system
(Vectris ${ }^{\circledR}$ SureScan $®$ with RestoreSensor ${ }^{\circledR}$ SureScan $尺$ implantable pulse generator (IPG) with AdaptiveStim $($ ) was implanted with leads spanning from the bottom of the T9 vertebral body to the mid portion of the T11 vertebral body (Fig. 1). The AdaptiveStim ${ }^{\circledR}$ setting allows the IPG to automatically adjust the stimulation amplitude as the patient changes position from standing to sitting to laying. The patient's device was programed to create paresthesias throughout the bilateral lower extremities from thighs to his feet. He denied any unpleasant paresthesias or areas of pain that were not covered. A summary of the spinal cord stimulator settings and parameters can be seen in Table 2.

After implantation the patient's symptoms improved by greater than $90 \%$. Equally important was the improvement in quality of life. In the follow-up period, he reported dramatic improvement in functional status,

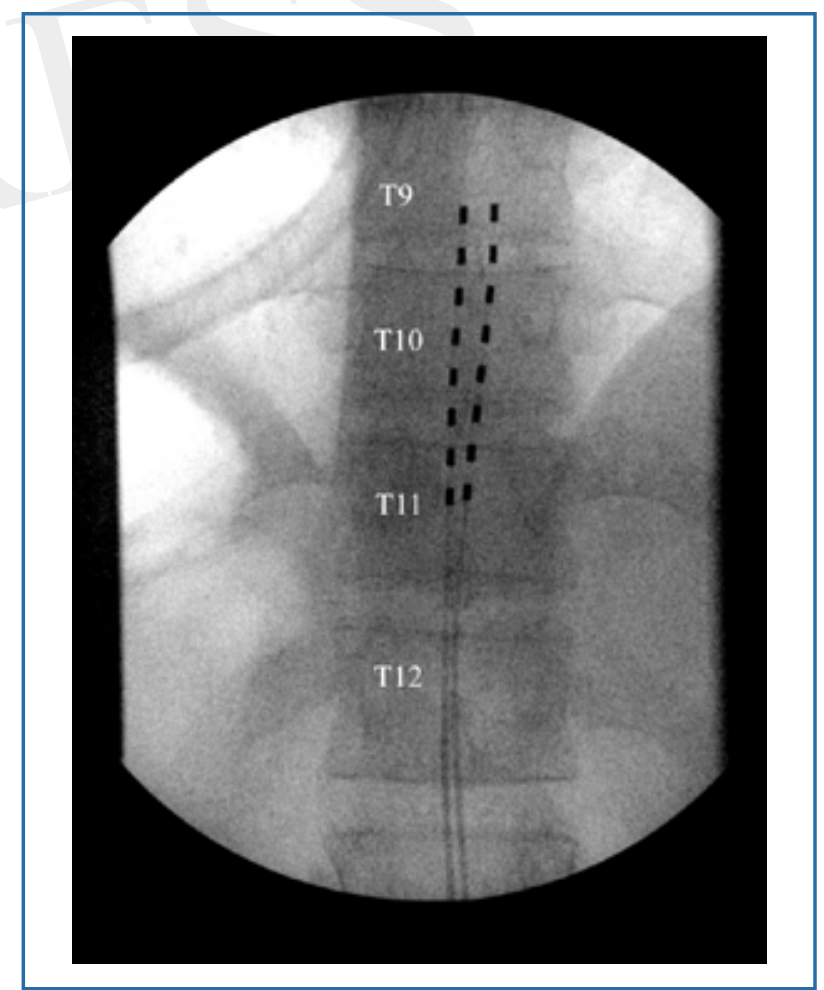

Fig 1. Anteroposterior (AP) fluoroscopic view of midline placed dual octet stimulator leads spanning the bottom of T9 to T11 post implantation. Central point of stimulation was obtained with the second and third contacts from the bottom of both leads. 
Table 2. Spinal cord stimulator settings.

\begin{tabular}{|l|c|c|}
\hline Amplitude Limits & Left & Right \\
\hline Upper & $10.5 \mathrm{~V}$ & $10.5 \mathrm{~V}$ \\
\hline Programmed & $2.55 \mathrm{~V}$ & $3.7 \mathrm{~V}$ \\
\hline Lower & $0.0 \mathrm{~V}$ & $0.0 \mathrm{~V}$ \\
\hline Upright Setting & $2.85 \mathrm{~V}$ & $2.75 \mathrm{~V}$ \\
\hline Mobile Setting & $2.55 \mathrm{~V}$ & $3.70 \mathrm{~V}$ \\
\hline Lying Back Setting & $1.20 \mathrm{~V}$ & $1.85 \mathrm{~V}$ \\
\hline Lying Right Side Setting & $1.65 \mathrm{~V}$ & $1.95 \mathrm{~V}$ \\
\hline Lying Left Side Setting & $0.0 \mathrm{~V}$ & $0.0 \mathrm{~V}$ \\
\hline
\end{tabular}

Contacts in use: Seconad and third from the bottom bilaterally. Frequency: $30 \mathrm{~Hz}$ Pulse width: $450 \mu \mathrm{s}$

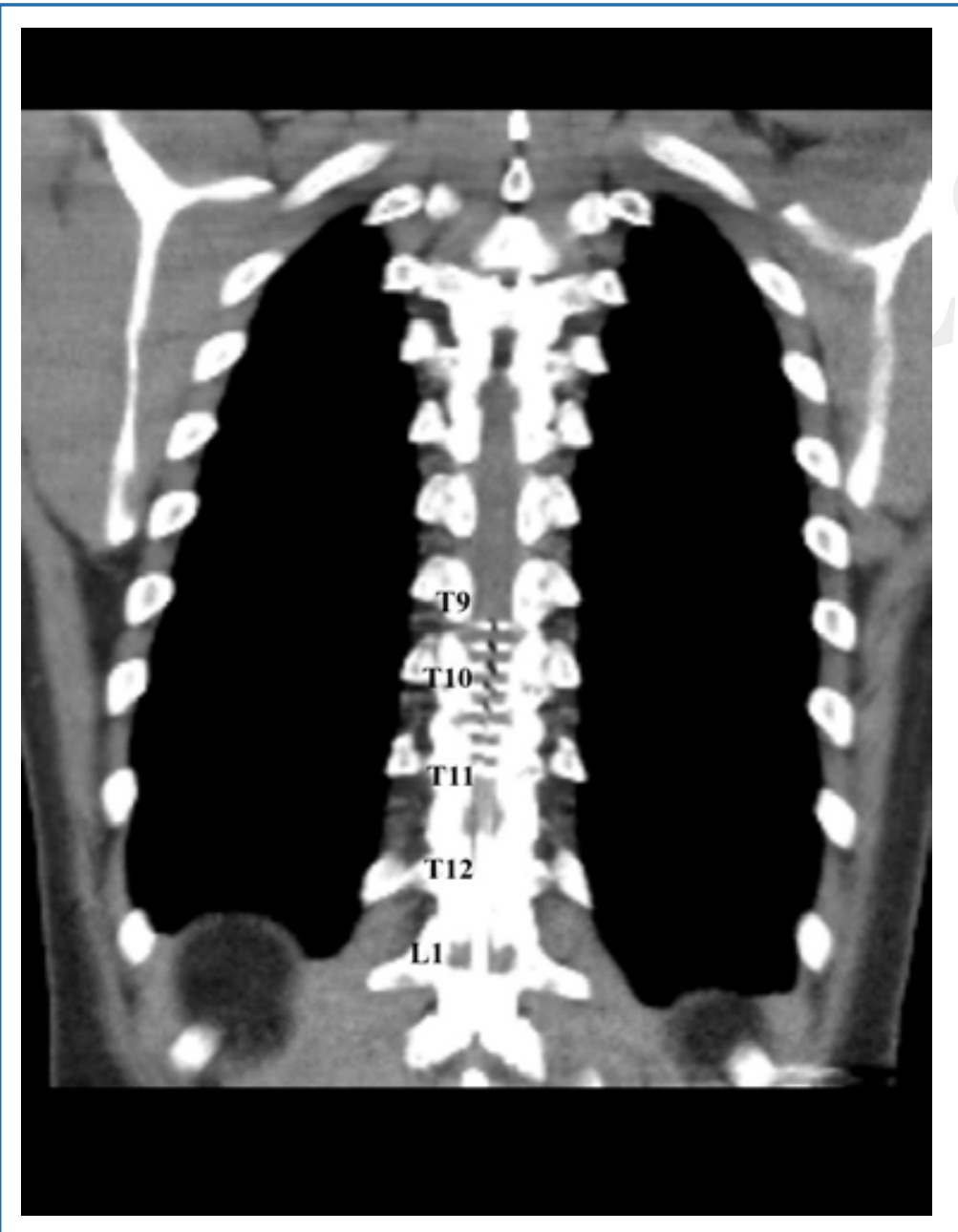

Fig. 2 Coronal view of computed tomography (CT) study obtained one year post implantation showing spinal cord stimulator leads in unchanged location spanning T9 to T11. cardiovascular activity, and focus on professional work. One year from implantation, the patient was employed in a full-time office position that required a 30-minute urban commute that included a mile of walking. Additionally, he was able to participate in weekly improvisational comedy performances, a passion hindered by his previous physical constraints. At the initial encounter he was not using pharmacologic analgesics, and on follow-up he continued to not require analgesic medications. One year after implantation, computed tomography for workup of unrelated gastrointestinal symptoms found the leads to be unchanged in position (Fig. 2).

\section{DISCUSSION}

Our patient described positive symptoms of pins and needles. Because the physical exam was normal, large-fiber neuropathy was deemed less likely. He had no autonomic symptoms and electromyography and MRI studies were normal. Because the patient had a history of vincristine chemotherapy, these findings lead to the diagnosis of chemotherapy-induced peripheral neuropathy.

Vincristine therapy has been associated with SFN in animal studies. In experimental animal models, painful neuropathies develop after vincristine treatment, with degeneration of the intraepidermal terminal arbors of sensory fibers and the activation of the skin's resident immune cells $(11,12)$.

The incidence of chemotherapyinduced peripheral neurotoxicity (CIPN) is currently unknown but has been reported to be about $40 \%$ (13). In one report, vincristine-induced peripheral neurotoxicity was as high as $80 \%$ in some small retrospective studies (9). Risk factors for the development of CIPN are shown in Table 3.

At first evaluation with a new primary care physician, the patient was referred to the pain clinic based on his function- 
ally limiting pain symptoms. The established relationship between the primary care physician and the pain clinic permitted timely referral and scheduling, minimizing delay in assessment and intervention by the team of board-certified pain specialists. Earlier referral to a pain specialist by the patient's oncologist, neurologist, and previous primary care physician may have led to a decreased period of suffering and more prompt return of function. Early consultation with a pain physician for complex pain syndromes may reduce patient exposure to parochial assessment and treatment.

While conventional spinal cord stimulation results in paresthesias that replace the painful sensation, the multiple specific mechanisms of dorsal column stimulation associated analgesia are yet fully elucidated. Spinal cord stimulation decreases the sensation of pain through modulating afferent nociceptive transmission at the dorsal horn via the gate control theory (14). According to this theory, a neuroanatomical gate in the dorsal horn of the spinal cord modulates the transmission of pain sensation centrally. A-beta activity at the dorsal horn inhibits c-fiber signal transmission, thereby "closing the gate." A-beta fiber triggering is more susceptible to electrical stimulation than that of $\mathrm{c}$-fiber triggering.

\section{REFERENCES}

1. Gregg EW, Gu Q, Williams D, de Rekeneire N, Cheng YJ, Geiss L, Engelgau M. Prevalence of lower extremity diseases associated with normal glucose levels, impaired fasting glucose and diabetes among U.S. adults aged 40 or older. Diabetes Res Clin Pract 2007; 77:485-488.

2. Beghi E, Monticelli ML. Chronic symmetric symptomatic polyneuropathy in the elderly: A field screening investigation of risk factors for polyneuropathy in two Italian communities. Italian General Practitioner Study Group (IGPST). J Clin Epidemiol 1998; 51:697-702.

3. Tavee JL, Zhou L. Small fiber neuropathy: A burning problem. Cleve Clin J Med 2009; 76:297-305.

4. Lacomis D. Small-fiber neuropathy. Muscle Nerve 2002; 26:173188.

5. Stewart JD, Low PA, Fealey RD. Distal small-fiber neuropathy: Results of tests of sweating and autonomic cardiovascular reflexes. Muscle Nerve 1992; 15:661-665.

6. Holland NR, Crawford TO, Hauer P, Cornblath DR, Griffin JW, McArthur JC. Small-fiber sensory neuropathies: Clinical course and neuropathology of idiopathic cases. Ann Neurol 1998; 44:47-59

7. Polydefkis M, Allen RP, Hauer P, Earley CJ, Griffin JW, McArthur JC. Subclinical sensory neuropathy in late-onset restless legs
Table 3. Risk factors for predicting chemotherapy-induced peripheral neurotoxicity.

- Longer duration of therapy/high cumulative dose

- Type of chemotherapeutic agent (e.g., vincristine, cisplatin, paclitaxel)

- Concurrent administration of other neurotoxic agents

- Pre-existing neuropathy (including chemotherapy-induced)

- Congenital diminished levels of dihydropyrimidine dehydrogenase

- Alcohol consumption, smoking, familial diabetes, elevated serum creatinine levels, and young age

\section{CONCLUSION}

Small fiber neuropathy after vincristine chemotherapy presents challenges for diagnosis and treatment. The associated pain symptoms may be mild or severe and may progress to significant disability. In this case, spinal cord stimulation provided an effective option for ameliorating the debilitating symptoms of small fiber neuropathy when comprehensive therapy failed. The patient progressively suffered for 7 years before being offered a trial of spinal cord stimulation. Interventional pain physicians should educate referring oncologists as to the potential for not only the treatment of cancer pain, but also on the options available for managing debilitating cancer treatment-related pain.

syndrome. Neurology 2000; 55:1115-1121.

8. Giuliani MJ, Stewart JD, Low PA. Distal small fiber neuropathy In: Low PA (ed). Clin Autonomic Disorders. 2nd ed. LippincottRaven, Philadelphia, 1997, pp 699-714.

9. Argyriou AA, Bruna J, Marmiroli P, Cavaletti G. Chemotherapyinduced peripheral neurotoxicity (CIPN): An update. Crit Rev Oncol Hematol 2012; 82:51-77.

10. Hurley RW, Adams MCB, Benzon HT. Neuropathic pain: Treatment guidelines and updates. Curr Opin in Anaesthesiol 2013; 26:580-587.

11. Uçeyler N, Kobsar I, Biko L, Ulzheimer J, Levinson SR, Martini R, Sommer C. Heterozygous PO deficiency protects mice from vincristine-induced polyneuropathy. J Neurosci Res 2006; 84:37-46.

12. Siau C, Xiao W, Bennett GJ. Paclitaxel- and vincristine-evoked painful peripheral neuropathies: Loss of epidermal innervation and activation of Langerhans cells. Exp Neurol 2006; 201:507514.

13. Wolf S, Barton D, Kottschade L, Grothey A, Loprinzi C. Chemotherapy-induced peripheral neuropathy: Prevention and treatment strategies. Eur J Cancer 2008; 44:1507-1515.

14. Melzack R, Wall PD. Pain mechanisms: A new theory. Science 1965; 150:971-978. 
